\title{
Design of Smart Devices For Older People: A User Centered Approach For The Collection Of Users' Needs
}

\author{
Silvia Imbesi ${ }^{1,}$ Giuseppe Mincolelli ${ }^{1}$ \\ ${ }^{1}$ Department of Architecture, University of Ferrara, Via della Ghiara 36, \\ 44121 Ferrara, Italy \\ \{giuseppe.mincolelli, silvia.imbesi\}@unife.it
}

\begin{abstract}
In this paper, it will be related a part of a design doctoral research that concerns the choice of the typology of users to analyzed to design smart devices for elderly people with physical symptoms related to gait and movement. The author will also explore which are the most advantageous ways to extrapolate from users' contributions their needs, that in User Centered Design are considered essential to elaborate the project requirements.
\end{abstract}

Keywords: User Centered Design · Design Research · Design For Older People - Design For PD Patients · User's Needs

\section{Global Ageing}

It is now generally acknowledged that the average age of the world population is constantly increasing and it will remain a constant trend for years to come, particularly in the most developed countries [1]. Without going into detail about the causes of this phenomenon, it is now necessary for our society to ponder how to face its consequences: one of the most obvious is the increase in public spending due to the shortage of economic and social institutional arrangements and policies that can guarantee a peaceful, dignified and healthy aging for the growing number of elderly people [2].

In order to reduce public welfare expenditures, it would be desirable to preserve older people's autonomy as long as possible, prolonging the stay in the own home and postponing the need of constant assistance and hospitalization. Increasing the quality of life of older people is a good strategy to not make them a burden for the health system as well as for their families [3].

\section{In-Home Care for Older People}

In-home senior care, in the absence of pathologies that do not allow it, is a good way to make aging cheaper, safer and more comfortable for the person [1]. Most of the people associate automatically the concept of in-home care to personal caregiving, thinking that the only way to facilitate the aged person's daily life in home is to have a qualified professional by his side taking on some tasks. 
Otherwise, it is important to recognize as fundamental part of in-home care even home ergonomics and different kinds of non-medical devices that can have a great impact on elderly's quality of life. Some symptoms of several pathologies can either be prevented or at least successfully accommodated, not to become debilitating in the short period, thanks to new technologies like the Internet of Things combined with Artificial Intelligence [4].

\section{User Centered Design for the Elderly}

Design Research has largely accepted the challenge of focusing on products and services for older people: in the last few years the number of researches and projects addressed to older people and their assistance and monitoring, is constantly increasing [5]. There are many design methodologies and approaches that can be used in this sector to combine properly the users' requirements with technologies' constrictions and opportunities, one of them, that the authors already used in several projects, is User Centered Design (UCD). UCD is recommended in particular to designers that are facing projects that require a high degree of usability to be successful [6], as design projects for older people. It allows the design of a product/service/process to be addressed to niche users, characterized by peculiarities that affect performances related to usability of products commonly used by most the population [7].

UCD focuses on shifting away from a reactive approach, in which environments, furniture and devices that cause problems and troubles are modified after something unpleasant happens, to a proactive approach that aims to design each element basing on the targeted requirements of a specific category of users [8].

The author is carrying on a doctoral research on User Centered methodologies to design smart devices which can help older people affected by Parkinson's Disease in reducing moving problems related to the gait and the walking: the final aim of this work is to analyze current technologies, inclusive methodologies, best practices and case studies, to create guidelines for the design of smart devices and services for the improvement of the autonomy of people that are starting to face the older age, living in their own home, frequently by themselves. The ongoing research is following the UCD approach, considered particularly suitable in the field of design for the silver age because it allows the categorization of users considering performances related to age restrictions, but is also extendable to limitations proper of a specific disease [6].

Following this methodology, it was decided to integrate the design process with the Quality Function Deployment, also known as QFD, a design tool that helps in the concept definition of products and services starting from users or costumers' needs and technological features of the product to be designed [9]. QFD is a graphic tool that collects in a matrix the list of the most important needs expressed by primary and secondary users, and an index of the most significant measurable characteristics and features of the product. Both needs and features are put in correlation through the matrix chart, this process, thanks to the QFD algorithm, helps designers evaluating the degree of relationship; starting from hierarchy resulting from expressed evaluations, it will be easier to decide the performances due to items or services to be designed [9]. 


\section{$4 \quad$ Collecting Needs of the Elderly}

The first stage of the UCD is the users' analysis, which aims at the detection of information about the selected categories of users, the designer will then process and combine information taken from the analysis to establish the project guidelines. The way information is gathered, the amount of data that is collected, the form in which the results of this collection are presented, are a significant part of the design choices of the project, affected by the designer's own attitude [8]. The choice of number and typology of the categories of users that will be analyzed, is as well part of the project.

In the previously mentioned doctoral research, the choice of users was considered particularly impactful on the project results; in this paper, it will be related the part of this research that concerns the choice of the typology of users that is going to be analyzed to design smart devices for elderly people with physical symptoms related to gait and movement, and how those users have been interviewed to extrapolate original and unexpected needs.

The users' analysis began with the study of dedicated literature, moving than to the direct observation of patients with Parkinson's Disease in public environments, and after to the observation of the users in their daily context.

\subsection{Definition of Primary Users, Secondary Users and Stakeholders}

In the Design Research context, we use to define as primary users the ones who directly use the product or service to achieve a goal; secondary users are people who use the product or service occasionally or mediated by other persons and lastly stakeholders that are people whose interests will be influenced by the product or people who have direct influence on the requirements of the system to be designed [10].

Assuming that older people at the early stages of Parkinson's Disease, still living in their own home without a professional caregiver, are the primary users of this project, it was noticed that the definition of secondary users and stakeholders wasn't as obvious as it might have seemed. The typology of product or service that is going to be designed highly influences the definition of who are the people that will get in touch with it and of what is their role in using the product. In this specific case, talking about devices that helps the gait and monitor the movements, it became obvious that the doctor who follows the patient has a most significant role than the relatives of the older person. Giving another example, talking about stakeholders, the health care institution that promotes the dissemination of devices that are not strictly medical has a greater impact on the product's system than those who directly sell it.

The final list of the users to be analyzed is the following:

- Primary user: older person affected by PD at the early stages of the disease, still living in his own house.

- Secondary users: consort of the primary user, doctors who are directly following the course of the disease of that specific person (geriatrician, neurologist, orthopedist...); occasional professional caregiver (carer for seniors, professional nurse...); relatives that occasionally take care of the person (they are profoundly different from the previous category due to the emotional implications); etc. 
- Stakeholders: health care institutions that promotes the dissemination of that kind of devices; commercial agents taking care of the promotion and sale of the product; health system workers who will have to cope with the degeneration of the pathology that has not been stemmed in time; etc.

\subsection{Analysis of Users' Needs}

The needs of the different users were collected in several ways: interviews, questionnaires, focus... but it was noticed that the best way to find hidden highlights and latent needs is the individual talk that does not follow a strict schedule. At this phase of the design process, after the definition of the categories of users, it can happen that the structure of the interviews is elaborated under the influence of preconceived ideas, of which the designer seeks confirmation when he faces the user. To remedy this potential problem that leads to a banalization of the outputs of the users' analysis, it is often useful to set aside the programmed schedule of the interview, and just focus on the inputs given by the person. We can imagine this action as a sort of brainstorming between the designer and the user, a sort of prequel of a codesign process. It is important to intend that brainstorming as a scientific one, not driven by randomness but rather structured as a group work method in which the creative play of the association of ideas is exploited: the aim is to bring out different alternative perspectives in order to identify and solve a problem.

In this specific project the choice was to set some questions related to the specific field of devices designed to help in solving troubles related to the gait of seniors affected by Parkinson's Disease. After each question, the interviewee was given only a few minutes to answer the question asked, during this time the interviewer identified two or three unexpected or curious elements on which it could be worthwhile to go into specific, investigating the reasons of these statements. Continuing the dialogue in this mode, the interview was much more interesting and rich of stimulating ideas than the previous ones conducted basing on a format conceived in the same way for all users without having yet known them.

\section{Conclusions}

At the current state of the doctoral research, it is possible to make two assessments regarding the advantages of the used methodology related to the User Centered Design approach.

Firstly, the early identification of a problem to be solved that was very specific led to a more informed and motivated choice in defining the various categories of users and their needs' hierarchization.

Secondly, the interpretation of the interview to the user as a brainstorming in a codesign process, rather than as a path guided by defined boundaries, has led to the enunciation of original and very personal needs that would surely not have emerged from the interviews conceived in a standard manner.

The adopted methodology is realistically applicable in other fields of the Design Research, in applied research projects that choose to use a Human Centered approach to solve a specific problem of a user with uncommon peculiarities. 


\section{References}

1. WHO World Health Organization: World Report on Aging and Health. WHO Press, Geneve (2015)

2. Beard JR, Biggs S, Bloom DE, Fried LP, Hogan P, Kalache A, et al. Introduction. In: Beard JR, Biggs S, Bloom DE, Fried LP, Hogan P, Kalache A, et al., editors. Global population ageing: peril or promise? Geneva: World Economic Forum (2012)

3. World Economic and Social Survey 2007: development in an ageing world. New York: United Nations Department of Social and Economic Affairs (2007)

4. Kelly, S.D.T., Suryadevara, N.K., Mukhopadhyay, S.C.: Towards the implementation of IoT for environmental condition monitoring in homes. IEEE Sens. J. 13(10), 3846-3853 (2013)

5. Coleman, R.: Improving the quality of life for older people by design. In: Graafmans, J., Taipale, V., Charness, N. (eds.) Gerontechnology: A Sustainable Investment in the Future. IOS Press, Amsterdam (1998)

6. Mao, J., Vredenburg, K., Smith, P., Carey, T.: The state of USER-CENTERED DESIGN PRACTICE UCD is gaining industry acceptance but its current practice needs fine-tuning. Commun. ACM 48(3), 105-109 (2005)

7. Mincolelli, G.: Customer/User centered design. Analisi di un caso applicativo, Maggioli, Rimini (2008)

8. ISO 13407: Human-centred design processes for interactive systems, ISO (1999)

9. Akao, Y.: Quality function deployment (QFD) integrating customer requirements into prduct design. Productivity Press, Portland (1990).

10. Preece, J., Rogers, Y., Sharp, H.,: Interaction Design, Apogeo Editore, Rovigo (2004) 